\title{
A cosmic equation of state for the inhomogeneous Universe: can a global far-from-equilibrium state explain Dark Energy?
}

\author{
Thomas Buchert \\ Arnold Sommerfeld Center for Theoretical Physics, Ludwig-Maximilians-Universität, \\ Theresienstraße 37, 80333 München, Germany \\ Email: buchert@theorie.physik.uni-muenchen.de
}

\begin{abstract}
A system of effective Einstein equations for spatially averaged scalar variables of inhomogeneous cosmological models can be solved by providing a 'cosmic equation of state'. Recent efforts to explain Dark Energy focus on 'backreaction effects' of inhomogeneities on the effective evolution of cosmological parameters in our Hubble volume, avoiding a cosmological constant in the equation of state. In this Letter it is argued that, if kinematical backreaction effects are indeed of the order of the averaged density (or larger as needed for an accelerating domain of the Universe), then the state of our regional Hubble volume would have to be in the vicinity of a farfrom-equilibrium state that balances kinematical backreaction and average density. This property, if interpreted globally, is shared by a stationary cosmos with effective equation of state $\mathbf{p}_{\text {eff }}=-1 / 3 \varrho_{\text {eff }}$. It is concluded that a confirmed explanation of Dark Energy by kinematical backreaction may imply a paradigmatic change of cosmology.
\end{abstract}

PACS numbers: 04.20.-q, 04.20.-Cv, 04.40.-b, 95.30.-k, 98.80.-Es, 98.80.-Jk

\section{Effective Einstein equations and the cosmic equation of state}

To set notation and to provide the framework for our argument, we recall a set of effective Einstein equations [2]. The argument presented can be carried over to studies of inhomogeneous cosmologies covering the Early Universe and radiation-dominated epochs with the help of the more general effective equations developed in 3 .

\subsection{Averaged equations}

For the sake of transparency we restrict ourselves to the matter model irrotational dust. Adopting a foliation of spacetime into flow-orthogonal hypersurfaces (which is possible for irrotational dust) with the 3-metric $g_{i j}$ in the line-element $d s^{2}=-d t^{2}+g_{i j} d X^{i} d X^{j}$, we define spatial averaging of a scalar field $\Psi$ on a domain $\mathcal{D}$ with volume $V_{\mathcal{D}}$ by:

$$
\left\langle\Psi\left(t, X^{i}\right)\right\rangle_{\mathcal{D}}:=\frac{1}{V_{\mathcal{D}}} \int_{\mathcal{D}} J d^{3} X \Psi\left(t, X^{i}\right),
$$


with $J:=\sqrt{\operatorname{det}\left(g_{i j}\right)}$, where $g_{i j}$ is the metric of the spatial hypersurfaces, and $X^{i}$ are coordinates that are constant along flow lines. Following [2] we define an effective scale factor through the volume of a simply-connected domain $\mathcal{D}$ in a hypersurface, normalized by the volume of the initial domain $\mathcal{D}_{\mathbf{i}}, a_{\mathcal{D}}:=\left(V_{\mathcal{D}} / V_{\mathcal{D}_{\mathbf{i}}}\right)^{1 / 3}$. Employing the fact that, for a restmass preserving domain $\mathcal{D}$, volume averaging of a scalar function $\Psi$ does not commute with its time-evolution, $\left\langle\partial_{t} \Psi\right\rangle_{\mathcal{D}}-\partial_{t}\langle\Psi\rangle_{\mathcal{D}}=\langle\Psi\rangle_{\mathcal{D}}\langle\theta\rangle_{\mathcal{D}}-\langle\Psi \theta\rangle_{\mathcal{D}}$, we can derive an effective equation for the spatially averaged expansion $\langle\theta\rangle_{\mathcal{D}}=\frac{\dot{V}_{\mathcal{D}}}{V_{\mathcal{D}}}=$ $3 \frac{\dot{a}_{\mathcal{D}}}{a_{\mathcal{D}}}=: 3 H_{\mathcal{D}}$ (with an effective Hubble functional $H_{\mathcal{D}}$ (an overdot denotes partial timederivative): setting $\Psi \equiv \theta$, inserting Raychaudhuri's evolution equation for $\partial_{t} \theta$ into the commutation rule above, and using the effective scale-factor $a_{\mathcal{D}}$ one obtains:

$$
3 \frac{\ddot{a}_{\mathcal{D}}}{a_{\mathcal{D}}}+4 \pi G\langle\varrho\rangle_{\mathcal{D}}-\Lambda=\mathcal{Q}_{\mathcal{D}} \quad ; \quad\langle\varrho\rangle_{\mathcal{D}}=\frac{M_{\mathcal{D}}}{V_{\mathcal{D}_{\mathrm{i}}} a_{\mathcal{D}}^{3}} .
$$

The first integral of (2) is directly given by averaging the Hamiltonian constraint:

$$
3\left(\frac{\dot{a}_{\mathcal{D}}}{a_{\mathcal{D}}}\right)^{2}-8 \pi G\langle\varrho\rangle_{\mathcal{D}}+\frac{\langle\mathcal{R}\rangle_{\mathcal{D}}}{2}-\Lambda=-\frac{\mathcal{Q}_{\mathcal{D}}}{2}
$$

where the total restmass $M_{\mathcal{D}}$, the averaged density, the averaged spatial Ricci scalar $\langle\mathcal{R}\rangle_{\mathcal{D}}$ and the kinematical backreaction term $\mathcal{Q}_{\mathcal{D}}$ are domain-dependent and, except the mass, time-dependent functions. The backreaction source term is given by

$$
\mathcal{Q}_{\mathcal{D}}:=2\langle I I\rangle_{\mathcal{D}}-\frac{2}{3}\langle I\rangle_{\mathcal{D}}^{2}=\frac{2}{3}\left\langle\left(\theta-\langle\theta\rangle_{\mathcal{D}}\right)^{2}\right\rangle_{\mathcal{D}}-2\left\langle\sigma^{2}\right\rangle_{\mathcal{D}}
$$

here, $I=\Theta^{i}{ }_{i}$ and $I I=\frac{1}{2}\left[\left(\Theta^{i}{ }_{i}\right)^{2}-\Theta^{i}{ }_{j} \Theta^{j}{ }_{i}\right]$ denote the principal scalar invariants of the expansion tensor, defined as minus the extrinsic curvature tensor, $-K_{i j}:=\Theta_{i j}$. In the second equality above it was split into kinematical invariants through the decomposition $\Theta_{i j}=\frac{1}{3} g_{i j} \theta+\sigma_{i j}$, with the rate of expansion $\theta=\Theta^{i}{ }_{i}$ and the rate of shear $\sigma^{2}=\frac{1}{2} \sigma_{i j} \sigma^{i j}$. (Note that vorticity is absent in the present gauge; we adopt the summation convention.)

The time-derivative of the averaged Hamiltonian constraint (3) agrees with the averaged Raychaudhuri equation (2) by virtue of the following integrability condition:

$\partial_{t} \mathcal{Q}_{\mathcal{D}}+6 H_{\mathcal{D}} \mathcal{Q}_{\mathcal{D}}+\partial_{t}\langle\mathcal{R}\rangle_{\mathcal{D}}+2 H_{\mathcal{D}}\langle\mathcal{R}\rangle_{\mathcal{D}}=0 \Leftrightarrow \partial_{t}\left(\mathcal{Q}_{\mathcal{D}} a_{\mathcal{D}}^{6}\right)+a_{\mathcal{D}}^{4} \partial_{t}\left(\langle\mathcal{R}\rangle_{\mathcal{D}} a_{\mathcal{D}}^{2}\right)=0$

\subsection{The cosmic quartet}

We may further introduce dimensionless average characteristics as follows [2]:

$$
\Omega_{m}^{\mathcal{D}}:=\frac{8 \pi G\langle\varrho\rangle_{\mathcal{D}}}{3 H_{\mathcal{D}}^{2}} ; \Omega_{\Lambda}^{\mathcal{D}}:=\frac{\Lambda}{3 H_{\mathcal{D}}^{2}} ; \Omega_{R}^{\mathcal{D}}:=-\frac{\langle\mathcal{R}\rangle_{\mathcal{D}}}{6 H_{\mathcal{D}}^{2}} ; \Omega_{Q}^{\mathcal{D}}:=-\frac{\mathcal{Q}_{\mathcal{D}}}{6 H_{\mathcal{D}}^{2}},
$$

where we have employed the effective Hubble-functional $H_{\mathcal{D}}$ introduced above that reduces to Hubble's function in the homogeneous-isotropic case. With these definitions the averaged Hamiltonian constraint (3) reads:

$$
\Omega_{m}^{\mathcal{D}}+\Omega_{\Lambda}^{\mathcal{D}}+\Omega_{R}^{\mathcal{D}}+\Omega_{Q}^{\mathcal{D}}=1
$$

providing a scale-dependent cosmic quartet relating all relevant "cosmological parameters". For $\mathcal{Q}_{\mathcal{D}}=0$ the above functionals reduce to the corresponding parameters of the standard homogeneous-isotropic models. 
The effective cosmological parameters defined in (6) can be considered to provide a fair representation of the values which also an observer would measure in a sufficiently shallow survey region $\mathcal{D}$ (the light-cone effect is not taken into account). We may therefore discuss estimates of those parameters in comparison with observed values. Note, however, that the interpretation of observations is mostly done by employing a standard Friedmannian cosmology as a 'fitting model' and therefore, geometrical inhomogeneities (that are hidden in the definition of the spatial averages in the Riemannian volume element, $c f$. Eq. (10) ) are ignored [6].

\subsection{The cosmic equation of state}

The above equations can formally be recast into standard zero-curvature Friedmann equations with new effective sources ([3]: Corollary 2)‡:

$\varrho_{\mathrm{eff}}^{\mathcal{D}}=\langle\varrho\rangle_{\mathcal{D}}-\frac{1}{16 \pi G} \mathcal{Q}_{\mathcal{D}}-\frac{1}{16 \pi G}\langle\mathcal{R}\rangle_{\mathcal{D}} \quad ; \quad \mathbf{p}_{\mathrm{eff}}^{\mathcal{D}}=-\frac{1}{16 \pi G} \mathcal{Q}_{\mathcal{D}}+\frac{1}{48 \pi G}\langle\mathcal{R}\rangle_{\mathcal{D}}$

$3 \frac{\ddot{a}_{\mathcal{D}}}{a_{\mathcal{D}}}=\Lambda-4 \pi G\left(\varrho_{\text {eff }}^{\mathcal{D}}+3 \mathbf{p}_{\text {eff }}^{\mathcal{D}}\right) ; 3 H_{\mathcal{D}}^{2}=\Lambda+8 \pi G \boldsymbol{\varrho}_{\text {eff }}^{\mathcal{D}} ; \dot{\boldsymbol{\varrho}}_{\text {eff }}^{\mathcal{D}}+3 H_{\mathcal{D}}\left(\boldsymbol{\varrho}_{\text {eff }}^{\mathcal{D}}+\mathbf{p}_{\text {eff }}^{\mathcal{D}}\right)=0$

Eqs. (9) correspond to the equations (2), (3) and (5), respectively. In these equations the kinematical backreaction term $\mathcal{Q}_{\mathcal{D}}$ itself obeys a stiff equation of state mimicking a dilatonic field in the fluid analogy (for further implications see [3]).

Given an equation of state in the form $\mathbf{p}_{\text {eff }}^{\mathcal{D}}=\beta\left(\varrho_{\text {eff }}^{\mathcal{D}}, a_{\mathcal{D}}\right)$ that relates the effective sources (8), the effective Friedmann equations (9) can be solved (one of the equations (9) is then redundant). Therefore, any question posed that is related to the evolution of scalar characteristics of inhomogeneous universe models may be "reduced" to finding the cosmic state on a given spatial scale. Although formally similar to the situation in Friedmannian cosmology, here the equation of state is dynamical and depends on details of the evolution of inhomogeneities. In general it describes non-equilibrium states.

\section{Explaining Dark Energy through kinematical backreaction}

The 'coincidence' that a Dark Energy source (modeled in the simplest case by a cosmological constant) starts to dominate around the epoch when also structure enters the non-linear regime suggests that there could be a physical relation between the effect of structure on the average expansion (known as backreaction effect) and the Dark Energy gap found in the Friedmannian standard model, thus providing a natural solution to this coincidence problem (besides the fact that more "exotic" explanations are then not needed).

The averaging problem in cosmology has a long history including calculations of the backreaction effect shortly after George Ellis [8] has pointed out its importance ([1]

$\ddagger$ Note that in this representation of the effective equations $\mathbf{p}_{\text {eff }}$ just denotes a formal "pressure": in the perfect fluid case with an inhomogeneous pressure function the foliation has to be differently chosen and there is a further averaged pressure gradient term [3]. 
and many works thereafter; references may be found in [7, 13, 14, 9, 5]). The new input into this discussion is the (not unsupported) claim by Edward Kolb et al. [13] that the condition (10) below could be satisfied within our regional Hubble volume, hence providing a smart explanation of the Dark Energy problem [15] without employing a cosmological constant, quintessence or corrections to Einstein's laws of gravity.

Setting $\Lambda=0$ in Eq. (2), the condition for an accelerating patch $\mathcal{D}$ of the Universe directly follows:

$$
\mathcal{Q}_{\mathcal{D}}>4 \pi G\langle\varrho\rangle_{\mathcal{D}} \quad ; \quad\langle\varrho\rangle_{\mathcal{D}}=\frac{M_{\mathcal{D}}}{V_{\mathcal{D}_{\mathbf{i}}} a_{\mathcal{D}}^{3}} \geq 0 .
$$

(With regard to (4), in order for $\mathcal{Q}_{\mathcal{D}}$ to be positive, expansion fluctuations would have to dominate over shear fluctuations.) This regional condition is weaker than the requirement of global acceleration, since it accounts for the regional nature of our observations. There is, however, a large body of opponents including myself who do not think that the condition (10) can be met within the standard picture of structure formation from CDM initial conditions. A number of caveats would have to be overcome related to explicit calculations of kinematical backreaction and observational constraints, which both will be discussed in more detail in a forthcoming paper [5. However, those caveats largely depend on assumptions that would consider perturbation theory on a Friedmannian background, would extrapolate (10) to the global scale according to the cosmological principle, and also would ignore the difficulties in relating the model parameters (6) to observations [9]. It is important to keep this disclaimer in mind in what follows. Restricting our attention to the universe model, as we shall do, there is no such caveat in our line of arguments.

Let us give an example that illustrates how strong the condition (10) appears, if we straightly compare the model parameters (6) with current observations. We first rewrite the condition (10) in terms of the dimensionless characteristics (6) $\oint$ :

$$
-\Omega_{Q}^{\mathcal{D}}>\frac{\Omega_{m}^{\mathcal{D}}}{4}
$$

We have to be aware that, if (11) holds on some large domain $\mathcal{D}$, which we may take to be as large as our observable Universe [13], then Hamilton's constraint in the form (71) also implies:

$$
\Omega_{\Lambda}^{\mathcal{D}}+\Omega_{R}^{\mathcal{D}}>1-\frac{3}{4} \Omega_{m}^{\mathcal{D}}
$$

showing that, for a low density parameter, we would need a substantial amount of negative curvature (positive $\Omega_{R}^{\mathcal{D}}$ ) in the inhomogeneous model (not in the 'fitting model') on the domain $\mathcal{D}$, if we put the cosmological constant equal to zero. (To reconcile a small curvature parameter in this condition with a non-vanishing cosmological constant would need an even larger value of $\Omega_{\Lambda}^{\mathcal{D}}$ than that suggested by the 'concordance model' of about 0.7.) The fact that a large value of kinematical backreaction goes along with a substantial amount of average Ricci curvature has also been stressed and discussed by $\S$ Note that, for a positive $\mathcal{Q}_{\mathcal{D}}, \Omega_{Q}^{\mathcal{D}}$ is, by definition, negative. 
Syksy Räsänen [14]. The condition (12), taken at face value (there are arguments why this could be naive), would contradict the widely agreed expectation that the curvature should be very small (the 'concordance model' assumes an exactly zero scalar curvature).

We, here, do not attempt to respectively verify or falsify the condition (10), but instead follow a line of arguments that assumes (10) to hold. That is to say, if kinematical backreaction can indeed account for the Dark Energy gap found in Friedmannian cosmology, then we have to closely examine implications of the above condition.

Let us first remark that the physical contents of (10) implies a strongly fluctuating cosmos, roughly speaking: fluctuations encoded in $\mathcal{Q}_{\mathcal{D}}$ have to be of the same order as the average density. This can only happen, if there is a strong coupling of kinematical backreaction to the averaged scalar curvature, even on the Hubble scale, for if this coupling is absent, (15) admits the special solution $\langle\mathcal{R}\rangle_{\mathcal{D}} \propto a_{\mathcal{D}}^{-2}$ and $\mathcal{Q}_{\mathcal{D}} \propto a_{\mathcal{D}}^{-6}([2]$ App.B), i.e. averaged scalar curvature behaves as in a constant-curvature Friedmannian model with effective scale factor $a_{\mathcal{D}}$, and fluctuations decay with the square of the (expanding) volume capturing what we may call cosmic variance. This particular solution mirrors what we would expect from standard cosmology. It implies that the averaged density $4 \pi G\langle\varrho\rangle_{\mathcal{D}} \propto a_{\mathcal{D}}^{-3}$ would substantially dominate over $\mathcal{Q}_{\mathcal{D}} \propto a_{\mathcal{D}}^{-6}$ in a globally expanding universe model after some time, even if we would start with the condition (10). Therefore, a strong coupling of $\mathcal{Q}_{\mathcal{D}}$ to $\langle\mathcal{R}\rangle_{\mathcal{D}}$ that changes the dependence on the effective scale factor sufficiently, is key to the explanation of Dark Energy through kinematical backreaction.

Notwithstanding, if we assume such a strong coupling exists in a realistic universe model, and if we suppose that our Hubble volume accelerates due to the fact that $\mathcal{Q}_{\mathcal{D}}$ dominates over $4 \pi G\langle\varrho\rangle_{\mathcal{D}}$, then we are entitled to say that a typical Hubble volume would correspond to a non-perturbative state in the vicinity of $\mathcal{Q}_{\mathcal{D}} \approx 4 \pi G\langle\varrho\rangle_{\mathcal{D}}$, i.e. it would not correspond to a perturbative state in the vicinity of a model with $\mathcal{Q}_{\mathcal{D}} \approx 0$, as expected in the standard picture of small perturbations of a Friedmannian background.

We are now going to identify this state in the effective equations. For this end let us now extend the spatial domain $\mathcal{D}$ to the whole Riemannian manifold $\Sigma$, which we assume to be compact. The cosmological principle would extrapolate the condition (10) to the global scale. However, since we are assuming a strongly fluctuating cosmos, it is more appropriate to allow for other Hubble volumes that are slightly decelarating with $\mathcal{Q}_{\mathcal{D}}<4 \pi G\langle\varrho\rangle_{\mathcal{D}}$, so that a typical Hubble volume would reside in a state close to the balance condition $\mathcal{Q}_{\mathcal{D}}=4 \pi G\langle\varrho\rangle_{\mathcal{D}} \|$. This balance condition furnishes an example for such a state. Extrapolating this condition to the global scale implies with (2) that the global effective acceleration vanishes, and together with (3) we face the global stationarity conditions:

$$
\mathcal{Q}_{\Sigma}=4 \pi G\langle\varrho\rangle_{\Sigma} \quad ; \quad\langle\mathcal{R}\rangle_{\Sigma}=12 \pi G\langle\varrho\rangle_{\Sigma}-6 H_{\Sigma}^{2} ; H_{\Sigma}=\frac{\mathcal{C}}{a_{\Sigma}}, \mathcal{C}=\text { const. }
$$

with the global kinematical backreaction $Q_{\Sigma}$, the globally averaged 3-Ricci curvature || In [5] a conservative estimate, based on current observational parameters, shows that such a cosmos provides room for at least 50 Hubble volumes. 
$\langle\mathcal{R}\rangle_{\Sigma}$, and the total restmass of the compact universe model $M_{\Sigma}$. The second condition above has been obtained by eliminating the backreaction term in (3) using the first condition. Eliminating instead the density source we can determine the constant $\mathcal{C}$ by evaluating (3) at the initial time: $\mathcal{C}^{2}:=\frac{1}{2} \mathcal{Q}_{\Sigma}\left(t_{i}\right)-\frac{1}{6}\langle\mathcal{R}\rangle_{\Sigma}\left(t_{i}\right)$. The cosmic equation of state for a stationary cosmos can be obtained by inserting (13) into (18). We find $\uparrow:$

$$
\mathbf{p}_{\text {eff }}^{\Sigma}=-\frac{1}{3} \varrho_{\text {eff }}^{\Sigma}
$$

Now, taking the time-derivative of the two conditions in (13) and employing restmass conservation on $\Sigma, \partial_{t}\langle\varrho\rangle_{\Sigma}+3 H_{\Sigma}\langle\varrho\rangle_{\Sigma}=0$, we obtain evolution equations for the global kinematical backreaction and the globally averaged Ricci curvature. These evolution equations are solved by:

$$
\mathcal{Q}_{\Sigma}=\frac{\mathcal{Q}_{\Sigma}\left(t_{i}\right)}{a_{\Sigma}^{3}} ;\langle\mathcal{R}\rangle_{\Sigma}=\frac{\langle\mathcal{R}\rangle_{\Sigma}\left(t_{i}\right)-3 \mathcal{Q}_{\Sigma}\left(t_{i}\right)}{a_{\Sigma}^{2}}+\frac{3 \mathcal{Q}_{\Sigma}\left(t_{i}\right)}{a_{\Sigma}^{3}}
$$

which indeed points to a strong coupling between kinematical backreaction and averaged scalar curvature: the rate of decay of $Q_{\Sigma}$ is in proportion to $\langle\varrho\rangle_{\Sigma}$ and can therefore be of the same order as $4 \pi G\langle\varrho\rangle_{\Sigma}$ today. The total kinematical backreaction $Q_{\Sigma} V_{\Sigma}=4 \pi G M_{\Sigma}$ is a conserved quantity. Actually, the solution (15) provides the first example of an exact solution of the effective Einstein equations with a non-trivial coupling of averaged scalar curvature to kinematical backreaction; (15) solves the integrability condition (5).

As in Friedmannian cosmology the fate of the effectively stationary inhomogeneous cosmos is determined by initial conditions, for the stationary effective scale factor $a_{\Sigma}=a_{S}+\mathcal{C}\left(t-t_{i}\right)$ can be restricted to a 'Big Bang model' by setting $a_{S}=0$, or it can emerge 10] from the effectively static cosmos $a_{S}=$ const., which is a subcase of the stationary one by setting $H_{\Sigma}=0 ; \mathcal{C}=0 \Leftrightarrow\langle\mathcal{R}\rangle_{\Sigma}\left(t_{i}\right)=3 \mathcal{Q}_{\Sigma}\left(t_{i}\right)$. The globally static inhomogeneous cosmos is characterized by the cosmic equation of state:

$$
\langle\mathcal{R}\rangle_{\Sigma}=3 \mathcal{Q}_{\Sigma}=\text { const. } \Rightarrow \mathbf{p}_{\mathrm{eff}}^{\Sigma}=\varrho_{\mathrm{eff}}^{\Sigma}=0
$$

\section{Concluding remarks}

We argued that an explanation of Dark Energy by kinematical backreaction effects and, hence, requiring the condition (10) to hold in our Hubble volume, implies a cosmos that is dominated by strong expansion fluctuations. We may speak of a far-from-equilibrium cosmic state in contrast to a perturbed Friedmannian state. We further argued that a system featuring strong fluctuations would conceivably, with some probability, create regional Hubble volumes in the vicinity of the stationary state (15). We have noted that, even if the Universe started in the vicinity of such a state, then it is important for the survival of the condition (10) that the evolution implies strong coupling between kinematical backreaction and averaged scalar curvature. We have shown that the globally stationary state indeed conserves strong fluctuations: the key-property is that

I It is interesting to compare this condition with the investigation of backreaction in inhomogeneous cosmon fields by Christof Wetterich [15], in particular with the 'cosmon equation of state'. 
$\mathcal{Q}_{\Sigma} \propto a_{\Sigma}^{-3} \propto\langle\varrho\rangle_{\Sigma}$ in large contrast to the case of a perturbed Friedmannian cosmos that would likely evolve into small fluctuations on the Hubble scale.

The picture that emerges entails a regionally fluctuating cosmos, where the region is as large as our Hubble volume. In fact this would imply a paradigmatic change of cosmology, since the properties seen on the Hubble scale are not extrapolated to the global scale as in Friedmannian cosmology. This remark is more evident, if a globally static model is envisaged: a globally static, but inhomogeneous cosmos is conceivable without employing a compensating cosmological constant in contrast to the classical Einstein cosmos. This mathematical possibility (put into perspective in [4]) would attain the status of a viable physical model, if the "classical" explanation of the Dark Energy problem in terms of kinematical backreaction effects were true. In such a cosmos the averaged scalar curvature is, for a non-empty Universe, positive; the global kinematical backreaction term takes the role of a positive cosmological constant. Regionally, such a cosmos features exponential gravitational instabilities, i.e. it has strong matter and curvature fluctuations.

In a forthcoming work [5] further implications are investigated. In particular, it is argued that, e.g. a globally static dust cosmos, provided it originates in this state, could be stabilized by backreaction and averaged curvature in contrast to the global stability properties of the classical Einstein cosmos [1]. It is further clarified that a fluctuation-dominated cosmos, expressed in a thermodynamic language, is in a far-from-equilibrium state compared with the Friedmannian "equilibrium state" by employing an entropy measure proposed in [12, which vanishes for Friedmannian cosmologies ("zero structure") and is positive and time-dependent for the globally stationary, but inhomogeneous cosmos.

This work was supported by the Sonderforschungsbereich SFB 375 'Astroparticle physics' by the German science foundation DFG. Special thanks go to Stéphane Colombi and Sabino Matarrese for stimulating discussions.

\section{References}

[1] J.D. Barrow, G.F.R. Ellis, R. Maartens and C.G. Tsagas, C. Q. G. 20, L155 (2003).

[2] T. Buchert, G. R. G. 32, 105 (2000).

[3] T. Buchert, G. R. G. 33, 1381 (2001).

[4] T. Buchert, in J. G. R. G. 9, Y. Eriguchi et al. (eds.), pp. 306-21 (2000).

[5] T. Buchert, to be submitted.

[6] T. Buchert and M. Carfora, Phys. Rev. Lett. 90, 031101-1-4 (2003).

[7] T. Buchert, M. Kerscher and C. Sicka, Phys. Rev. D. 62, 043525 (2000).

[8] G.F.R. Ellis, in General Relativity and Gravitation (Reidel Pub., Dordrecht), pp. 215-88 (1984).

[9] G.F.R. Ellis and T. Buchert, Phys. Lett. A., in press; gr-qc/0506106 (2005).

[10] G.F.R. Ellis and R. Maartens, C. Q. G. 21, 223 (2004).

[11] T. Futamase, M.N.R.A.S. 237, 187 (1989).

[12] A. Hosoya, T. Buchert and M. Morita, Phys. Rev. Lett. 92, 141302-1-4 (2004).

[13] E.W. Kolb, S. Matarrese and A. Riotto, submitted to Phys. Rev. D.; astro-ph/0506534 (2005).

[14] S. Räsänen, submitted to Phys. Rev. D.; astro-ph/0504005 (2005).

[15] C. Wetterich, Phys. Rev. D 67, 043513 (2003). 